\title{
Prevalencia de infección por Chlamydia trachomatis en prostitutas registradas de la ciudad de Durango, México
}

\author{
Cosme Alvarado-Esquivel, M.C., D r. en C., ${ }^{(1)}$ Alfonso García-Villanueva, M.C., ${ }^{(2)}$ \\ D ora Elia C astruita-Limones, Lic. en Enf., Q .F.B., (1) Francisco Javier Cardosa-N evárez, M.C., (2) \\ Rosario Ruiz-Astorga, Q .F.B..$^{(1)}$
}

\begin{abstract}
Alvarado-Esquivel C, García-Villanueva A, Castruita-Limones DE, Cardosa-Nevárez FJ, Ruiz-A storga R. Prevalencia de infección por Chlamydia trachomatis en prostitutas registradas de la ciudad de Durango, México. Salud Publica Mex 2000;42:43-47.
\end{abstract}

\begin{abstract}
Resumen
Objetivo. Determinar la prevalencia de infección por Chlamydia trachomatis en prostitutas registradas de la ciudad de Durango, Durango y establecer si existe alguna correlación entre los datos epidemiológicos y la infección. Material y métodos Fueron estudiadas 247 prostitutas y se obtuvieron muestras endocervicales y datos epidemiológicos. La prueba Chlamydiazyme (A bbott Laboratories, EUA) fue usada para detectar el antígeno de C. trachomatis. Resultados Fueron positivas para C. trachomatis 41 prostitutas (16.6\%), y 37 de ellas habían tenido actividad sexual en diferentes estados de la República mexicana, en comparación con las 206 mujeres negativas, entre las que sólo 109 habían tenido relaciones sexuales fuera de Durango $(p<0.0001)$. El nivel socioeconómico bajo fue más frecuentemente observado en prostitutas $C$. trachomatis positivas $(39 / 41,95.1 \%)$ que en las negativas $(171 / 206,83 \%)(p=0.05)$. Conclusiones Encontramos una prevalencia de infección por C. trachomatis de $16.6 \%$, la cual se asoció a una actividad sexual de las prostitutas en diversas entidades federativas, y se pudo observar que el padecimiento tiende a presentarse más frecuentemente en mujeres de un nivel socioeconómico bajo.
\end{abstract}

Palabras clave: infecciones por Chlamydia; prevalencia; prostitución; México

\author{
Alvarado-Esquivel C, García-Villanueva A, \\ Castruita-Limones DE, Cardosa-Nevárez FJ, \\ Ruiz-Astorga R. \\ Prevalence of Chlamydia \\ trachomatis infection in registered prostitutes \\ of Durango City, Mexico. \\ Salud Publica Mex 2000;42:43-47.
}

\begin{abstract}
A bstract
Objective. To determine the prevalence of Chlamydia trachomatis infection among registered prostitutes of Durango City and to establish whether there is a correlation between epidemiological factors and infection. Material and methods. Two-hundred-and-forty-seven registered prostitutes of Durango city were studied. Endocervical samples and epidemiolo gical data were obtained. C. trachomatis antigen was detected with the Chlamydiazyme test (Abbott Laboratories, USA.) Results. Forty-one (16.6\%) out of 247 prostitutes were positive to $C$. trachomatis. Thirty-seven out of the 41 positive women had had sexual activity on several States of Mexico (95.1\%), as compared to only 109 out of 206 negative women $(53.0 \%) \quad(p<.0001)$. Prostitutes positive to $C$. trachomatis $(39 / 41,95.1 \%)$ were more likely to belong to low socioeconomic level than negatives (171/206, $83 \%)(p=0.05)$. Conclusions. The prevalence of $\mathrm{C}$. trachomatis infection was $16.6 \%$. C. trachomatis infection was associated with sexual activity in multiple States of Mexico, and had a tendency to be associated with low socioeconomic level.
\end{abstract}

Key words: Chlamydia infections; prevalence; prostitution; Mexico

(1) Instituto de Investigación Científica, Universidad Juárez del Estado de Durango, México.

(2) Dirección Municipal de Salud Pública y Medio A mbiente, Durango, México.

Fecha de recibido: 16 de julio de 1999 - Fecha de aprobado: 9 de noviembre de 1999

Solicitud de sobretiros: Dr. Cosme Alvarado Esquivel. Departamento de Inmunología y Biología Molecular. Instituto de Investigación Científica, Universidad Juárez del Estado de Durango. Avenida Universidad y Fanny A nitua, 34000 Durango, D urango, México. 
C hlamydia trachomatis es una bacteria gramnegativa patógena, transmitida frecuentemente por vía sexual. ${ }^{1-3}$ Las infecciones se caracterizan por la presencia de uretritis o cervicitis, ${ }^{1,4}$ y son más comunes en adultos jóvenes, especialmente entre aquellos en condiciones socioeconómicas bajas y entre mujeres con múltiples compañeros sexuales. Además, pueden conducir a complicaciones graves y costosas como abscesos pélvicos, enfermedad pélvica inflamatoria, esterilidad y dolor pélvico crónico, entre otras. ${ }^{5-7}$ Hay evidencia de que C. trachomat is puede causar muertes neonatales, ${ }^{6}$ abortos, ${ }^{8}$ ruptura de membranas y trabajo de parto prematuro. ${ }^{9,10}$ En niños nacidos de madres infectadas, $35 \%$ desarrolla conjuntivitis, y 20\%, neumonía. ${ }^{1,7}$ Diversos serotipos de $C$. trachomatis han sido descritos. Las infecciones por los serotipos D-K se asocian a enfermedades como uretritis, cervicitis, conjuntivitis y neumonia. ${ }^{11}$

El estándar de oro para el diagnóstico de infección por C. trachomatis ha sido el cultivo celular acompañado de inmunofluorescencia. Sin embargo, esta prueba no está disponible en la mayoría de los laboratorios; por lo tanto, frecuentemente se recurre a ensayos inmunoenzimáticos y a otro tipo de métodos, a pesar de su menor sensibilidad y especificidad. ${ }^{11-14}$

La prevalencia de infección por $C$. trachomatis entre mujeres dedicadas a la prostitución en varias ciudades del mundo, exceptuando a la ciudad de México, varía entre 19.8 y 38.3\%. ${ }^{15-23}$ En México, un estudio realizado en ciudades de Michoacán, Puebla, Veracruz e Hidalgo notificó una prevalencia de $12.9 \%$ en $1990 .^{24}$ En la ciudad de México se han señalado prevalencias de $11.1 \%$ en $1993,{ }^{25}$ y de $11.7 \%$ en $1994,{ }^{24}$ con un aumento de $13 \%$ en 1995 y a $20 \%$ en los años 1996 y $1997 .{ }^{26}$ En un estudio reciente realizado en el estado de Guerrero, se encontró una prevalencia de $36.9 \% .{ }^{27}$ Debido a la falta de datos en Durango, se decidió realizar este estudio y así determinar, mediante la prueba inmunoenzimática, la prevalencia de infección cervical por C. trachomatis en prostitutas registradas de la ciudad de Durango; asimismo, se buscó establecer si existe alguna correlación entre los datos epidemiológicos y la infección por C. trachomatis.

\section{Material y métodos}

En la ciudad de Durango la prostitución se ejerce únicamente con permiso de la Dirección Municipal de Salud Pública y Medio Ambiente. Utilizamos el término "prostituta registrada" (PR) para referirnos a las mujeres que se dedican a la prostitución con permiso sanitario. Esa oficina vigiló a 551 PR durante 1997 y 1998, años en los que se realizó este estudio; de ellas, 247 ejercieron la prostitución de manera activa y acudieron regularmente a sus exámenes sanitarios; el resto era una población irregular, que migraba frecuentemente. Posiblemente existían actividades clandestinas del sexo comercial femenino; sin embargo, podrían ser mínimas.

En este estudio incluimos a las 247 PR regulares antes mencionadas, después de haber obtenido su consentimiento informado. Personal médico aplicó a cada mujer un cuestionario para conocer datos sociodemográficos y epidemiológicos como: edad, estado civil, lugar de nacimiento, antecedentes ginecológicos y obstétricos; antecedentes de enfermedades transmitidas sexualmente; uso de anticonceptivos y de preservativos, así como frecuencia de ruptura del mismo; tiempo de promiscuidad sexual y número de compañeros por semana; uso de drogas, alcoholismo; actividades sexuales en otros estados de la República mexicana y en el extranjero, y nivel socioeconómico. Estas características fueron consideradas variables categóricas. El nivel socioeconómico se estimó usando los criterios de Bronfman. ${ }^{28}$

De cada mujer se obtuvo una muestra endocervical, para luego ser analizada en el Instituto de Investigación Científica de la Universidad Juárez del Estado de Durango, Durango, donde se detectó el antígeno de C. trachomatis mediante la prueba inmunoenzimática Chlamydiazyme (Abbott Laboratories, IL, Estados Unidos de América-EUA). Doscientos microlitros de una suspensión de buffer y muestra se colocaron en un pozo de reacción, al igual que el control positivo y el negativo. Se agregó una perla con anticuerpos anti-C. trachomatis y se le incubó a $37{ }^{\circ} \mathrm{C}$ durante una hora. Después de un lavado con agua destilada, se agregaron 200 microlitros de anticuerpos anti-C. trachomatis y se les incubó a $37^{\circ} \mathrm{C}$, también durante una hora. Se eliminaron los anticuerpos no unidos mediante lavado y se incubaron las perlas con un conjugado de anticuerpo-enzima contra el anticuerpo específico. Tras otro lavado se transfirieron las perlas a tubos de reacción y se incubaron por 30 minutos con O-fenildiamina, deteniendo la reacción con $1 \mathrm{ml}$ de ácido sulfúrico. Las muestras se procesaron en el analizador Quantum II (Abbott Diagnostics, EUA) y se determinó la absorbancia a una longitud de onda de $492 \mathrm{~nm}$.

Como herramienta para el análisis estadístico de los datos, se utilizó el Software InStat Versión 2.0. Para el análisis estratificado de variables categóricas, se calcularon las razones de momios (RM) con intervalos de confianza de $95 \%$, y la significancia fue analizada por medio de la prueba exacta de Fisher de dos colas. El tamaño de la muestra por cada grupo fue de 
31, el cual da $80 \%$ de poder, una RM mínima significativa de 4 , y $5 \%$ de nivel de significancia.

\section{Resultados}

De las 247 mujeres estudiadas, 41 (16.6\%) fueron positivas a la prueba inmunoenzimática. Las PR estudiadas tenían una edad de 18 a 50 años ( $\bar{X} 29.7 \pm$ DE 7.8). Doscientas diez mujeres (85\%) pertenecían a un nivel socioeconómico bajo, y 37, (15\%) a un nivel medio. Ciento ochenta y nueve de ellas $(76.5 \%)$ eran originarias del estado de Durango, 57 (23.1\%) eran de otros 21 estados de la república, y una $(0.4 \%)$, de Guatemala. Ciento noventa y una prostitutas $(77.4 \%)$ eran solteras; 10 (4.0\%) vivían en unión libre; $22(8.9 \%)$ eran divorciadas; $14(5.7 \%)$, viudas, y $10(4.0 \%)$ estaban casadas. Doscientas veintinueve $(92.7 \%)$ prostitutas usaron algún tipo de método anticonceptivo: 141 (57.1\%), hormonales; 49 (19.8\%), salpingoclasia, y $39,(15.8 \%)$ dispositivo intrauterino. Respecto al uso del condón, $244(98.8 \%)$ prostitutas dijeron utilizarlo en cada relación sexual, y tres (1.2\%) señalaron no usarlo; $41(16.6 \%)$ mujeres refirieron la ruptura ocasional del condón.

El número de embarazos por PR fue de 0 a 15 (mediana 3); de partos de 0 a 11 (mediana 3); de cesáreas, de 0 a 3 (mediana 1), y, abortos de 0 a 4 (mediana 1). El número de compañeros sexuales por semana fue de 1 a 60 (mediana 4). La mayoría 197/247 (79.8\%) de las PR refirió tener de 1 a 5 compañeros por semana. Treinta y cuatro de 41 positivas a la prueba de $C$. trachomatis (83\%) pertenecían a este grupo. Las mujeres estudiadas habían ejercido la prostitución de 1 a 25 años ( $\bar{X} 3.93 \pm D E 4.34)$. De las 41 positivas a C. trachomatis, 34 (83\%) tenían una antigüedad de 1 a 5 años; cinco (12.2\%); de entre 6 y 10 años, y dos (4.8\%); de más de 10 años. Veinticuatro mujeres (9.7\%) declararon haber utilizado algún tipo de droga inhalada o habérsele aplicado por vía intravenosa. Además, 191 (77.3\%) prostitutas consumían regularmente bebidas alcohólicas, al menos una vez cada semana.

Treinta y siete $(15 \%)$ prostitutas manifestaron haber padecido alguna enfermedad de transmisión sexual, incluyendo gonorrea $(23 / 37,62.2 \%)$, sífilis $(8 / 37$, $21.6 \%)$ y tricomoniasis $(6 / 37,16.2 \%)$. Ciento cuarenta y cinco $(58.7 \%)$ mujeres dijeron viajar frecuentemente por el territorio nacional, a fin de trabajar sexualmente en otros estados, y 40 (16.2\%) refirieron viajes al extranjero con el mismo propósito. El Cuadro I muestra un resumen de las variables estudiadas y los resultados de la prueba de $C$. trachomatis.

Todas las $41 \mathrm{PR}$ positivas regresaron por su resultado y se les dio tratamiento con Azitromicina (1 gr do- sis única). A nueve se les repitió el ensayo dos meses después. Dos de ellas persistieron positivas y siete resultaron negativas.

\section{Discusión}

En este estudio se encontró, entre las PR de la ciudad de Durango, una prevalencia de infección por C. trachomatis de $16.6 \%$, la cual es más baja que la notificada en prostitutas de la ciudad de México (20\%, en 1997), ${ }^{26}$ y de otras partes del mundo, ${ }^{15-23}$ y es más alta que la prevalencia registrada en Michoacán, Puebla, Veracruz e Hidalgo (12.9\% en 1990). ${ }^{24}$ Sin embargo, esta comparación debe tomarse con reservas, ya que se utilizaron métodos diagnósticos diferentes. El método inmunoenzimático tiene las siguientes ventajas: factibilidad de realización en laboratorios que no cuentan con la experiencia o infraestructura para realizar el cultivo, menos requisitos para el transporte del espécimen y estandarización de tecnología. La principal desventaja es que la sensibilidad y la especificidad son inferiores al estándar de oro ( 85.4 y $99.2 \%$, respectivamente, en muestras endocervicales).

Los factores de riesgo para infección por $C$. trachomatis notificados en la literatura científica y especializada incluyen la promiscuidad sexual, el no uso de condón, la utilización de drogas intravenosas, un estado socioeconómico bajo, el antecedente de enfermedades de transmisión sexual y el uso de dispositivos intrauterinos. ${ }^{6,24,29} \mathrm{Al}$ analizar estos factores de riesgo en nuestro estudio, observamos que la prevalencia de infección por $C$. trachomatis en usuarias de condón fue de $16 \%$, mientras que en las no usuarias fue de $67 \%$ $(p=0.07)$. La prevalencia de infección por $C$. trachomatis en las PR de nivel socioeconómico bajo fue de $18.6 \%$, en tanto que en las de nivel medio fue de $5.4 \%$ ( $p=0.05)$, lo que apoya marginalmente la existencia de una relación inversa entre la infección por $C$. trachomatis y el nivel socioeconómico. ${ }^{1,30}$

Además, en este estudio observamos una alta asociación entre actividades sexuales en otros estados del país fuera de Durango, e infección por C. trachomatis en PR (RM=6.4; IC95\% 2.4-17.0, $p<0.0001)$. Esta variable deberá ser analizada en otros estudios. La asociación fue observada en las mujeres que acudieron regularmente a control sanitario, por lo que podríamos esperar una mayor prevalencia de infección por C. trachomatis en PR que no acuden constantemente a sus revisiones debido a que migran con mayor frecuencia que las PR regulares. De igual manera, la prevalencia de infección por $C$. trachomatis en prostitutas no registradas y que no siguen el control sanitario, pudiera ser también alta. 


\section{Cuadro I \\ Relación entre las características de las PR y la infección por C. trachomatis. Ciudad de Durango, Durango, MÉxico, 1997-1998}

\begin{tabular}{|c|c|c|c|c|c|}
\hline \multirow[b]{2}{*}{ Característica } & \multicolumn{3}{|c|}{ No. (\%) de PR } & & \\
\hline & $\begin{array}{l}\text { Total } \\
(n=247)\end{array}$ & $\begin{array}{l}\text { Prueba positiva para C. trachomatis } \\
\qquad(n=41)\end{array}$ & $\begin{array}{l}\text { Prueba negativa para C. trachomatis } \\
\qquad(n=206)\end{array}$ & RM & IC95\% \\
\hline ETS & $37(14.9)$ & $5(12.2)$ & $32(15.5)$ & 0.8 & $0.3-2.1$ \\
\hline
\end{tabular}

Antecedentes obstétricos

\begin{tabular}{|c|c|c|c|c|c|}
\hline Embarazos & $236(95.5)$ & $39(95.1)$ & $197(95.6)$ & 0.9 & $0.2-4.3$ \\
\hline Partos & $214(86.6)$ & $35(85.4)$ & $179(86.9)$ & 0.9 & $0.3-2.3$ \\
\hline Cesáreas & 44 (17.8) & $9(22.0)$ & $35(17.0)$ & 1.4 & $0.6-3.1$ \\
\hline Abortos & 78 (31.6) & $15(36.6)$ & $63(30.6)$ & 1.3 & $0.6-2.6$ \\
\hline
\end{tabular}

\begin{tabular}{|c|c|c|c|c|c|}
\hline Uso de drogas & $24 \quad(9.7)$ & $3(7.3)$ & $21(10.2)$ & 0.7 & $0.2-2.5$ \\
\hline Actividad en otros estados & $145(58.7)$ & $36(95.1)$ & $109(53.0)$ & 6.4 & $2.4-17.0$ \\
\hline Actividad en el extranjero & $40(16.2)$ & $4 \quad(9.8)$ & $36(17.5)$ & 0.5 & $0.2-1.5$ \\
\hline Uso de anticonceptivos & $229(92.7)$ & $36(87.8)$ & $193(93.7)$ & 0.5 & $0.2-1.4$ \\
\hline No uso de condón & $\begin{array}{ll}3 & (1.2)\end{array}$ & $2(4.9)$ & $1 \quad(0.5)$ & 10.5 & $0.9-118.9$ \\
\hline Ruptura de condón & 41 (16.6) & $5(12.2)$ & $36(17.5)$ & 0.7 & $0.2-1.8$ \\
\hline
\end{tabular}

Nivel socioeconómico

\begin{tabular}{|c|c|c|c|c|c|}
\hline Bajo & $210(85.0)$ & $39(95.1)$ & $171(83.0)$ & 4 & $0.9-17.3$ \\
\hline Medio & $37(15.0)$ & $2(4.9)$ & $35(17.0)$ & 0.3 & $0.1-1.1$ \\
\hline Alcoholismo & $191(77.3)$ & $31(75.6)$ & $160(77.7)$ & 0.9 & $0.4-2.0$ \\
\hline
\end{tabular}

Lugar de nacimiento

\begin{tabular}{lrrrrr} 
Estado de Durango & $189(76.5)$ & $30(73.2)$ & $159(77.2)$ & 0.8 & $0.4-1.7$ \\
\hline O tros estados & $58(23.5)$ & $11(26.8)$ & $47(22.9)$ & 1.2 & $0.6-2.7$
\end{tabular}

RM: razón de momios

ETS: enfermedades transmitidas sexualmente

Aunque podría esperarse que a mayor tiempo de ejercicio de la prostitución, mayor frecuencia de infección por C. trachomatis, nuestros resultados mostraron una tendencia hacia lo contrario. Esto podría probablemente explicarse por la mayor frecuencia de contactos sexuales durante los primeros años de ejercicio de la prostitución o porque las PR se iniciaron en esta actividad en una edad más joven. De hecho, la infección clamidial en México se ha correlacionado de manera elevada con la edad joven. ${ }^{30}$

Los resultados de este trabajo permiten proponer acciones para el control de la infección por C. trachomatis, así como recomendar que las prostitutas se realicen rutinariamente exámenes para detectar esta facteria, principalmente aquellas que tienen actividades como sexoservidoras en varios estados de la república y un bajo nivel socioeconómico. Finalmente, sería recomendable monitorear la respuesta al tratamiento en las prostitutas infectadas.

\section{Referencias}

1. Divo A. Microbiología médica. 4a. edición. México, D.F.: Interamericana, 1990:251-257.

2. Brooks GF, Butel SB, 0 rnston LN . Microbiología médica. 15a. edición. México, D.F.: El Manual Moderno, 1997:363-373.

3. Kingsbury DT,W ager GE, Segal GP. Microbiología médica. México, D.F.: Limusa, 1989:231-235.

4. Jensen $M M, W$ right $D N$. Introducción a la Microbiología médica. México, D.F.: Prentice Hall Hispanoamérica, 1987:275-380.

5. Santaella GA, Valdez O E, Díaz HJ. Chlamydia trachomatis cervical. Incidencia en población mexicana de alto riesgo (resumen). G inecol 0 bstet Mex 1994;62 supl 1:65. 
6. Bowien W R, Holmes KK. Chlamydia trachomatis (tracoma, infecciones perinatales, linfogranuloma venéreo y otras infecciones genitales). En: Mandell GL, Douglas RG, Benett JE, ed. Enfermedades infecciosas (Principios y práctica). 3a edición. Buenos Aires: Médica Panamericana, 1991: 1502-1517.

7. Guardiano CA. Bacteriología clínica. En: Iovine E,A tilo SA. El laboratorio en la clínica. Metodología analítica, fisio patología e interpretación semiológica. 3a edición. Buenos Aires: Médica Panamericana, 1991:1123-1127. 8. Díaz BG , D íaz LE, Servín RF. Frecuencia de Chlamydia trachomatis en el cérvix de pacientes embarazadas en control prenatal. Ginecol 0 bstet Mex 1997;65:48-52.

9. Flores MF, Durazo Q F, Unda FE, Flores OF, Morfin MJ, Ramírez BG. Infecciones por Chlamydia trachomatis en el Hospital SantaTeresa (resumen). Ginecol 0 bstet Mex 1994;62 supl I:67.

10. Echaniz AG, C alderon JE, C arnalla BN, Soto N A, C ruz VA, Gatica MR. Prevalencia de infección cervicovaginal por Chlamydia trachomatis en población femenina de la ciudad de Cuernavaca, Morelos, México. Salud Publica Mex 1992;34:301-307.

11. Hahn H, Falke D, Kaufmann SHE, U IImann U. Medizinische Mikrobiologie und Infektiologie. 3a edición.Alemania:Springer-Verlag, 1999:441-450. 12. Finegold SM, Baron EJ. Diagnóstico microbiológico. $7 a$ edición. Argentina: Médica Panamericana, 1989;505-510.

13. Koneman EW, Allen SD, D owellVR, Sommers H M. Diagnóstico microbiológico. 3a edición. Argentina: Médica Panamericana 1992: 820-822.

14. Black CM. Current methods of laboratory diagnosis of Chlamydia trachomatis infections. Clin Microbiol Rev 1997;1:160-184.

15. A lary M, Laga M, Vuylsteke B, N zila N, Poit P. Signs and symptoms of prevalent and incident cases of gonorrhea and genital Chlamydia infection among female prostitutes in Kinshasa, Zaire. C lin Infect D is 1996;22: 477-484.

16. Vuylsteke B, Laga M, Alary M, Gerniers M M, Lebughe JP, N zyla N . Clinical algorithms for the screening of women for gonococcal and Chlamydia infection: Evaluation of pregnant women and prostitutes in Zaire. C lin Infect $D$ is 1993;17:82-88.

17. Kaptue L, Zekeng L, D joumessi S, Monny LM, N ichols D, D ebuysscher R. HIV and Chlamydia infections among prostitutes in Yaounde, C ameroon. Genitourin Med 1991;67:143-145.

18. N kya W M, Gillespie SH, Howlett W, Elford J, N yamuryekunge C, Assenga $\mathrm{C}$. Sexually transmitted diseases in prostitutes in Moshi and Arusha, N orthern Tanzania. Int J STD A IDS 1991;2:432-435.
19. Prins M, Hooykaas C, C outinho RA, Van D oornum GJ,Van Den Hoek A). Incidence and risk factors for acquisition of sexually transmitted diseases in heterosexuals with multiple partners. Sex Transm D is 1994; 21: 258-267.

20. Ertem $E$, Dereli $D$, Serter $D$, Ergin 0 . D etection of Chlamydia trachomatis in prostitutes working in a brothel in Izmir. Mikrobiyol Bul 1993; 27:335-337.

21. Ichinose M,Amano Y, KobayashiY, Matsue T, 0 no gawaT, Saito M. Epidemiological study on Chlamydia trachomatis infection in obstetrics and gynecology field in Tokio. Kansenshogaku Zasshi 1991;65:1309-1316.

22. Ramachandran S, N geow YF. The prevalence of sexually transmitted diseases among prostitutes in Malaysia. G enitourin Med 1990;66:334-336. 23. Venegas VS, Villafranca P, Madrid JP, Cosenza H, Bygdeman S. Gonorrhoea and urogenital Chlamydia infection in female prostitutes in Tegucigalpa, Honduras. Int J STD AIDS 1991;2:195-199.

24.Valdespino GJ, García GM, C hiriboga C, C ruz PC, Loo ME, López SA. Las enfermedades de transmisión sexual y la epidemia deVIH/SIDA. Salud Publica Mex 1995:37:549-555.

25. U ribe $F$, Hernández $M, C$ onde $C$, Juárez L, Allen $B$, A naya $R$ et al. Low prevalences of HIV infection and sexually transmitted disease among female commercial sex workers in Mexico city.Am J Public Health 1997;87: 1012-1015.

26.Vázquez A,Cruz C,Vázquez R. Prevalencia de Chlamydia trachomatis en población con prácticas de riesgo, un estudio de 3 años (resumen). Enferm Infecc Microbiol 1998;18:S40.

27. Leyva MA, Rodríguez MA, Martínez N E,Antonio V,Alarcón LC, Flores E. Chlamydia trachomatis en sexoservidoras del estado de Guerrero (resumen). XXII Congreso N acional de Q uímica Clínica 1999; MSTL 21.5.

28. Bronfman M, Guiscafré $H, C$ astro V, C astro R, Gutiérrez G. La medición de la desigualdad: una estrategia metodológica, análisis de las características socioeconómicas de la muestra. Arch Invest Med 1988;19:351-360.

29. Cartwright PS. Enfermedad inflamatoria pélvica. En: Jones HW, Colston W A, Burnett CL, ed. Tratamiento de ginecología de N ovak. México, D.F.: Interamericana Mc Graw Hill, 1991:449-456.

30.A costa-C azares B, Ruiz-Maya L, Escobedo-D e la Peña J.Prevalence and risk factors for Chlamydia trachomatis infection in low-income rural and suburban populations of Mexico. Sex Transm D is 1996;23: 283-288. 